\title{
Performance Evaluation of Microgrid Management Function in KERI pilot plant
}

\author{
Jong-Yul Kim, Jin-Hong Jeon and Seul-Ki Kim \\ New and Renewable Energy Research Center \\ Korea Electrotechnology Research Institute \\ 70 Boolmosangil, Changwon, 641-120 (Korea) \\ Phone/Fax number:+0082 055 2801336, e-mail: jykim@keri.re.kr, jhjeon@keri.re.kr, blksheep@keri.re.kr
}

\begin{abstract}
As usual, the microgrid operates in grid-connect mode, but, when a fault occurs in the upstream grid, it should disconnect and shift into islanded operation mode. In gridconnect mode, the controlling power flow at PCC is necessary management function rather than the frequency and voltage. However, the controlling the frequency and voltage is asked in islanded mode. To achieve these management goals appropriately, the cooperative control strategy among microsources is needed. This supervisory control action is executed at MMS. In this paper, the cooperative control strategy of microgrid components is presented and the management performance is evaluated by using microgrid pilot plant. The pilot plant consists of the PV, PV/Wind Hybrid system, BESS, diesel generators, and RLC loads, which are connected to $380 \mathrm{~V}$ low voltage lines. The test results show that the proper control strategy can ensure stability of microgrid in islanded mode and maintain the power flow of PCC at desired value successfully.
\end{abstract}

\section{Key words}

Microgrid, Co-operative Control, MMS, Pilot Plant, DGs.

\section{Introduction}

Though the penetration of distributed generations to the electric power system is limited due to the lack of economical benefits, it will be accelerated for various reasons. The increase in DGs (Distributed Generators) penetration depth and the presence of multiple DGs in electrical proximity to one another have brought about the concept of the microgrid [1]-[3], which is a cluster of interconnected DGs, loads and intermediate energy storage systems. As usual, the microgrid operates in gridconnect mode, but, when a fault occurs in the upstream grid, it should disconnect and shift into islanded operation mode. In grid-connect mode, the frequency of the microgrid is maintained within a tight range by the main grid. Therefore, the controlling power flow at PCC (Point of Common Coupling) is necessary management function rather than the frequency and voltage. In an islanded operation, however, which has relatively few microsources, the local frequency control of the microgrid is not straightforward. The frequency of the power system has a strong coupling with the active power in the network. The rate of change of frequency depends on the inertia of the systems. During a disturbance, the frequency of the microgrid may change rapidly due to the low inertia present in the microgrid. Therefore, local frequency control is one of the main issues in islanded operation [4]. To achieve the management goals in two different operation modes, the cooperative control strategy of all components is needed.

In this paper, the cooperative control strategy of microgrid components is presented and evaluated performance of management functions by a hardware test using pilot plant.

\section{Configuration of Microgrid}

A microgrid is a cluster of interconnected micro sources that are referred to as distributed generators, loads and intermediate energy storage systems that co-operate with each other to be collectively treated by the grid as a controlled load or generator [5]. Fig. 1 shows a typical configuration of microgrid. It comprises, in addition to loads, RES (Renewable Energy Sources) as well as a diesel engine, gas engine, micro turbine and an energy storage system as support systems to fulfil the stable operation of a facility. This microgrid is connected to the grid at the PCC, and operates in parallel with a utility grid under normal situations. The microgrid disconnects from the utility grid, however, and transfers into islanded operation mode when a fault occurs in the upstream grid.

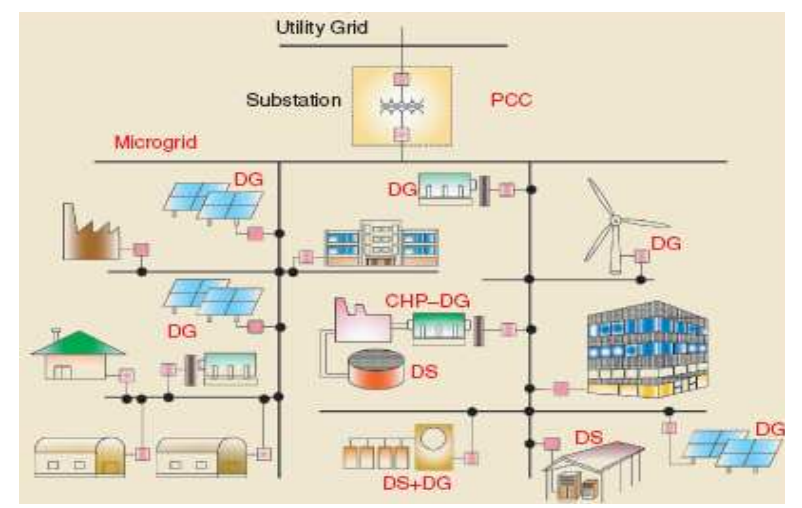

Fig. 1. Typical configuration of microgrid. 
The conventional micro sources existing in the distribution network mainly use rotating machines. They are directly connected the grid to supply electric power, but new technologies, such as micro turbine, PV, Wind, and Fuel Cells, that are proposed for use in the microgrid are not suitable for supplying energy to the grid directly. They have to be interfaced with the grid through an inverter stage. The intermediate ESS (Energy Storage System) is an inverter-interfaced battery bank (BESS), SMES, supercapacitor or flywheel. The storage device in the microgrid is analogous to the spinning reserve of large generators in the conventional grid. They ensure the balance between energy generation and consumption especially during islanded operation [6].

The microgrid has a hierarchical control structure as shown in Fig. 2. It has two control layers: MMS (Microgrid Management System) and LC (Local Controller). The MMS is a centralized controller that deals with management functions such as disconnection and re-synchronization of the microgrid and the load shedding process. In addition to this function, the MMS is responsible for the supervisory control of micro sources and the energy storage system. Using collected local information, the MMS generates a power output set point and provides it to the LCs. An LC is a local controller that is located at each micro source and controls the power output according to the power output set from the MMS [7].

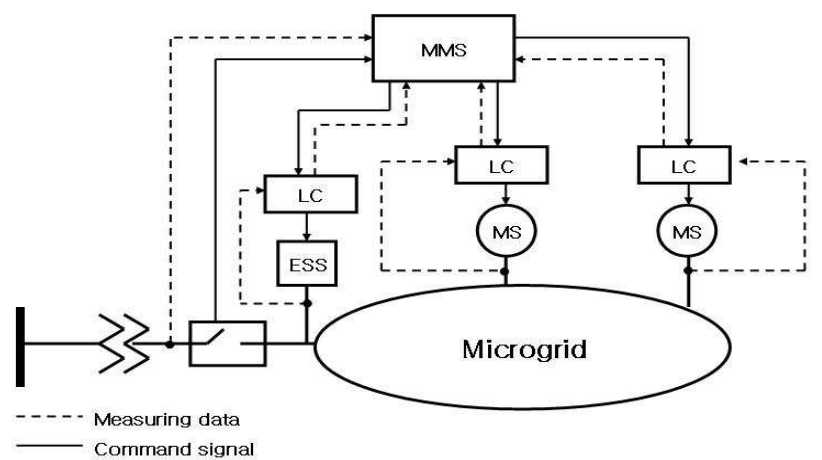

Fig. 2. The hierarchical control structure of microgrid.

\section{Microgrid Operation Management}

The microgrid can operate both in grid-connected and in stand-alone mode. Its functionalities as well as its control structure depend on the mode of operation.

\section{A. Grid-Connected Mode}

In grid-connected mode balance between the generation and the consumption is guaranteed by the utility grid. And also, the frequency and voltage of the microgrid are maintained within a tight range by the main grid. In addition, the microgrid is considered as a dispatchable aggregated generating plant or load by system operator.

The system operator can asks microgrid to control the power flow at PCC as a certain constant level for peak shaving at peak time or solving congestion of distribution network. Therefore, the controlling the power flow at PCC is needed rather than frequency and voltage control.
For controlling the power flow at PCC, MMS should control the power output of Microsources but RES cooperatively. The MMS keep monitoring the states of microgrid and then dispatching the power output set point of microsources. By controlling the power at the PCC, the microgrid operates as a constant power generator or a constant power load.

\section{B. Islanded Mode}

Islanded mode can be took place by unplanned faults at an upstream grid. When the fault occurs, MMS should detect the event as soon as possible and disconnect the microgrid from the upstream grid. In islanded mode, which has relatively few micro sources, the local frequency control of the microgrid is not straightforward.

During islanding, the power balance between supply and demand does not match at the moment. As a result, the frequency of the microgrid will fluctuate, and the system can experience a blackout unless there is an adequate power balance matching process. The control of the energy storage system inverter responds in milliseconds. Otherwise, because of the nature of the control of some micro sources such as the diesel generator, gas engine, and fuel cell, these micro sources have a relatively slow response time. Obviously, the cooperative control of ESS and other microsources should be needed to maintain the frequency and voltage of the microgrid.

\section{Control strategy for Microgrid}

\section{A. Grid-Connected Mode}

In grid-connected mode, all of the microsources adopt the fixed power control, which means that the micro sources and ESS generate constant active and reactive power. Therefore, MMS can handle the power output of each microsource to carry out the necessary management function. The MMS can receive the information about system states by communication. The closed loop control issues power output set-point over the communication channel to each microsource. The LCs ultimately are responsible for regulating the power flow locally in each component. The MMS compares the measured power flow at PCC and the reference value to obtain the error.

This error is regulated by regulator in MMS. The total required power command is applied to the dispatch function to generate a power output set-point for each individual available controllable microsource. This regulation action is executed every second by MMS.

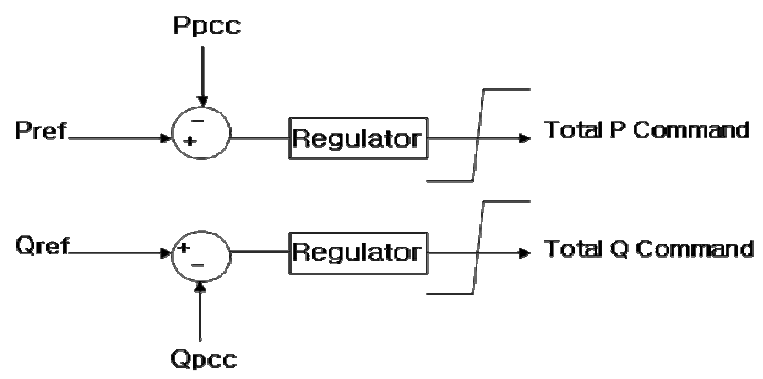

Fig. 3. Control scheme for regulating power flow at PCC. 


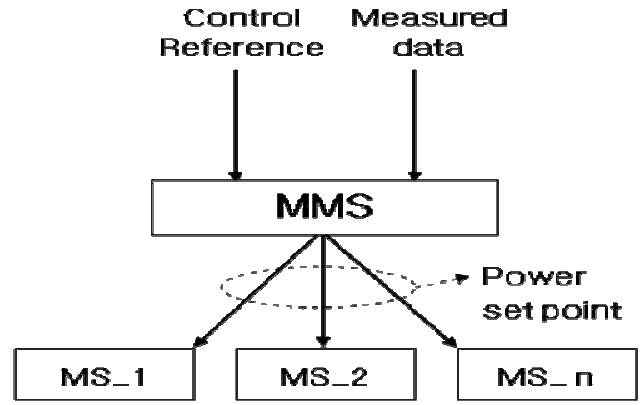

Fig. 4. Cooperative control strategy in grid-connected mode.

\section{B. Islanded Mode}

In grid-connect operations, all of the micro sources and ESS adopt the fixed power control mode. Usually, power output of the ESS may be fixed at zero when the microgrid is operated in grid connect mode, but, since the fixed power control of ESS supplies constant power, it cannot provide proper frequency and voltage controlling ability in islanded operation. Therefore, the control scheme of the ESS has to be switched from fixed power control to frequency/voltage control during islanded operation. Otherwise, other controllable micro sources are still fixed power control. By proper control action of the ESS, the frequency and voltage of the microgrid can be brought back to the normal values after a disturbance.

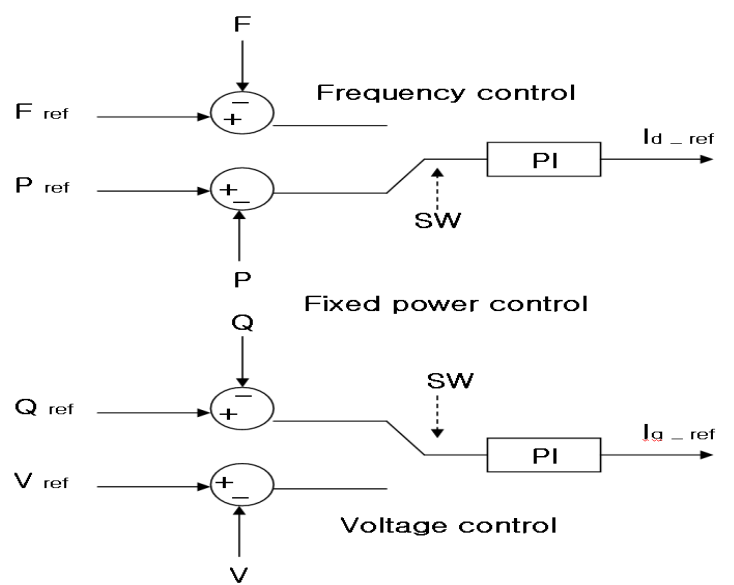

Fig. 5. Control scheme of the ESS.

Though the frequency and the voltage of microgrid in islanded operation can be effectively controlled by applying an F/V control scheme in the ESS, the control capability of the ESS may be limited by its available energy storage capacity. Therefore, the power output of the ESS should be brought back to zero as soon as possible. The secondary regulation control is in charge of returning the current power output of the ESS to the preplanned value, which is usually set at zero. This secondary regulation control is performed by using cooperative control scheme of micro sources and ESS in MMS. When operating in islanded mode, the ESS regulates the frequency and the voltage in local control actions, and then the MMS calculates the proper power outputs of each micro source to make the power output of the ESS equal zero, as shown in Fig. 6. The final set points are calculated every second by MMS. The final reactive power output set point produced can also be calculated in the same way as active power.

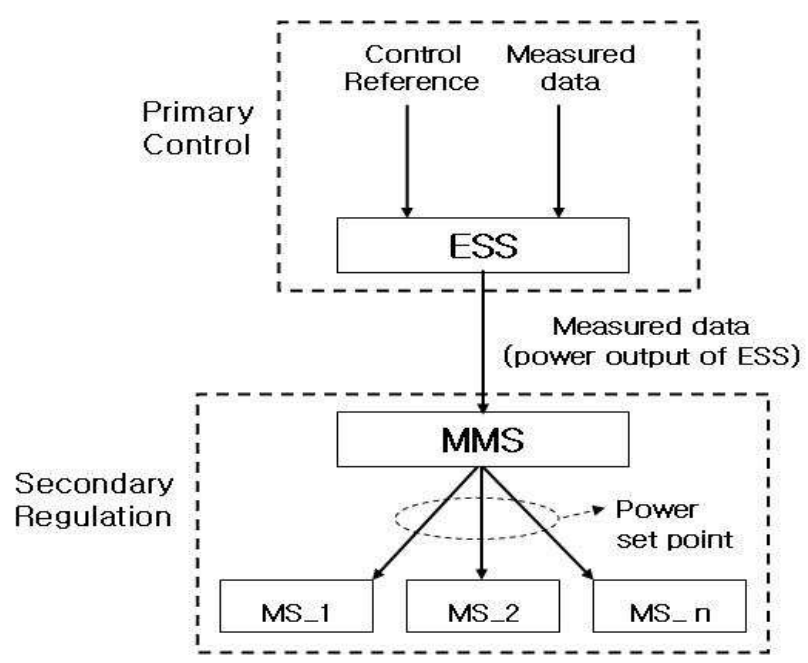

Fig. 6. Cooperative control strategy in islanded mode.

\section{Feature of KERI Microgrid Pilot Plant}

Fig. 7 and Fig. 8 show configuration and feature of a $120 \mathrm{~kW}$ microgrid pilot plant been constructed in Korea Electro-technology Research Institute (KERI) and feature of each components in microgrid pilot plant.

The PV, PV/Wind Hybrid system, BESS, and diesel generators have been connected to $380 \mathrm{~V}$ low voltage lines, which connects to a $22.9 \mathrm{kV}$ distribution feeder through a pole transformer. The STS is also connected to $380 \mathrm{~V}$ busbar which is near the PCC to disconnect microgrid from utility grid when the fault occurs. All components are installed and being operated now. The plant specification is as follows:

1) Test System Configuration

-No. of Controllable Source : 2 (Diesel generators)

- No. of Renewable Source : 2 (PV, H/B)

- No. of Energy Storage System : 1 (BESS)

- No. of Loads : 3

2) Generation Capacity of Microsources

- PV : $10 k W$

- Diesel generator : $70 \mathrm{~kW}(50 \mathrm{~kW}+20 \mathrm{~kW})$

- H/B : 20kW (Wind 10kW+PV 10kW)

3) Capacity of Energy Storage System - BESS (Battery Energy Storage System) 10kWh

- PCS of BESS system 25kVA

4) Load

- Peak Load : 99kW+j44kVar

- Constant impedance model $(R / X)$

Fig. 9 shows the developed prototype MMS. It is a PCbased equipment and designed for management of small scale microgrid pilot plan. It controls microgrid components by RS-485 serial communication methods. 


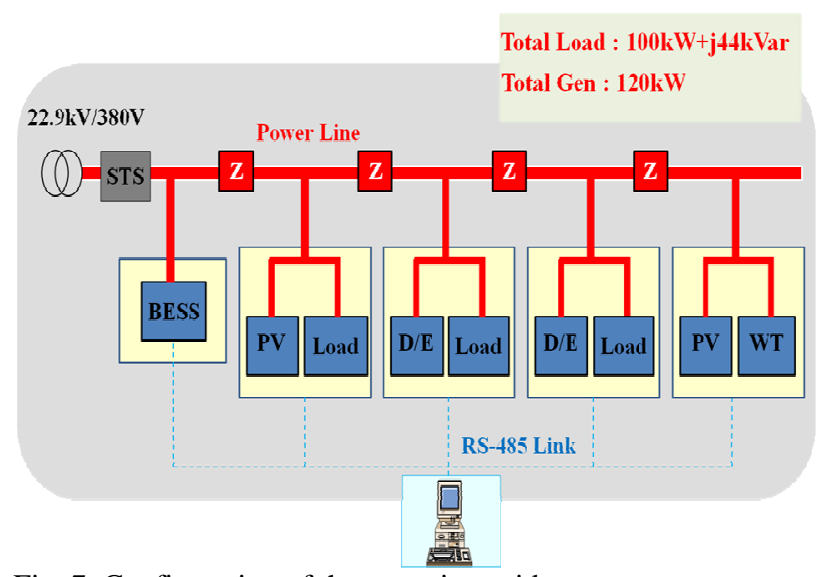

Fig. 7. Configuration of the test microgrid system.

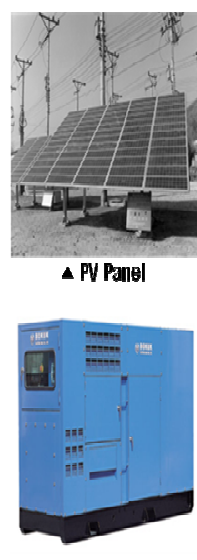

4 Dlesel Generator
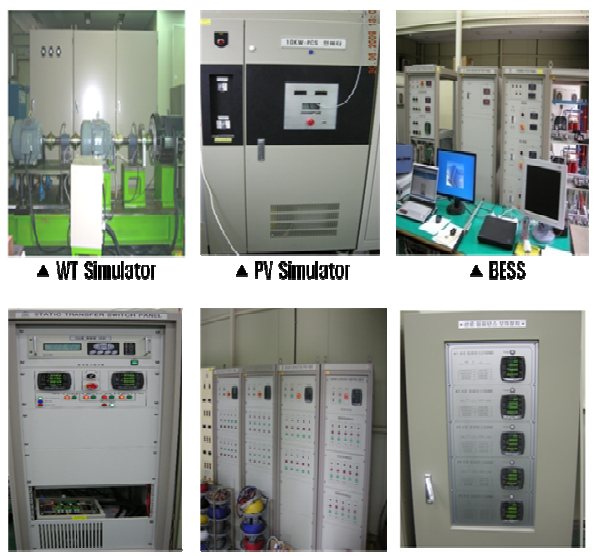

$\triangle$ STS/ED

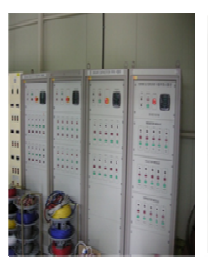

$\triangle$ RLC Load

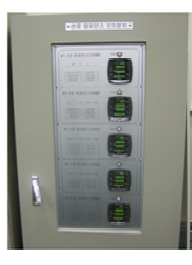

$\Delta$ line Simulator
Fig. 8. Feature of the components in microgrid pilot plant.

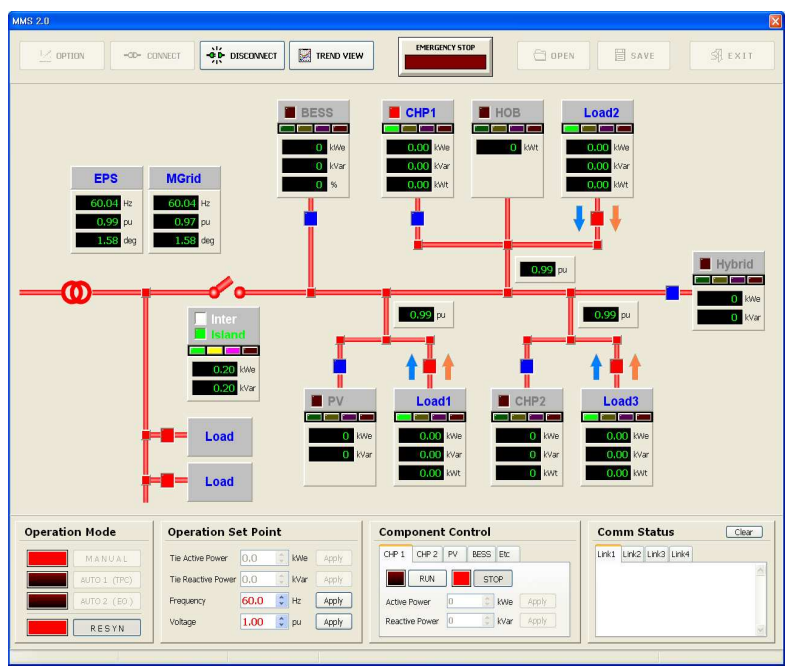

Fig. 9. Feature of developed MMS.

\section{Test Results}

The performance of microgrid management functions are evaluated by hardware test using pilot plant. The dynamic behaviours of the microgrid with two different operating modes are tested. During the test period, the load and power outputs of PV and H/B are varying in accordance with the time as shown in Fig. 10 and Fig. 11.

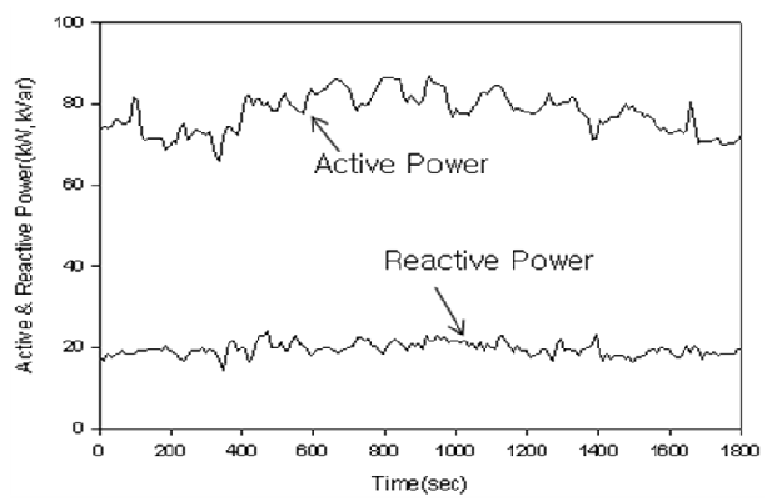

Fig. 10. Load fluctuation during test period.

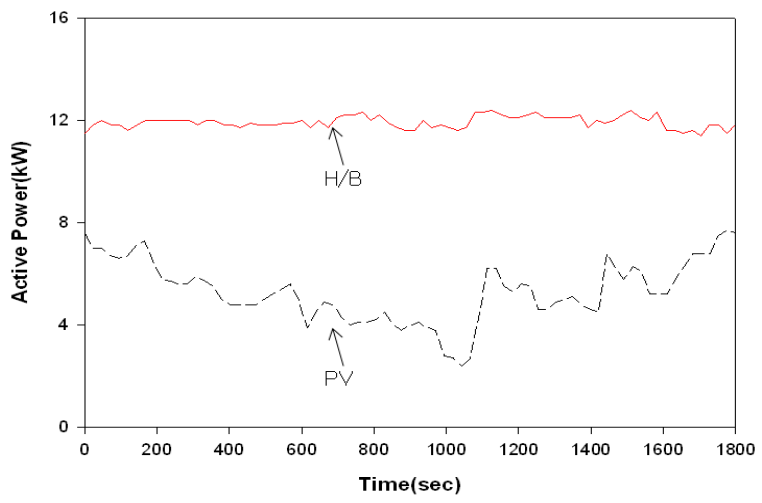

Fig. 11. Power outputs of H/B and PV.

\section{A. Grid-Connected Mode}

In this operating mode, the power flow at PCC is controlled. The MMS receives the microgrid state information via RS-485 link and calculates the power set points of two diesel generators. In this case, the reference values of power flow at PCC set to be $20 \mathrm{~kW}+\mathrm{j} 0 \mathrm{kVar}$. The control action of MMS tries to make the power flow at PCC be a $20 \mathrm{~kW}+\mathrm{j} 0 \mathrm{kV}$ ar by controlling the diesel engine generators as shown in Fig 12 and Fig. 13. As a result of this regulating action of MMS, the average of power flow at PCC is regulated in accordance with the reference values as shown Fig. 14. The test results show that microgrid can acts as a aggregated constant load or generator. This means that the distribution operator can utilize the microgrid to achieve the effective system operation goals such as peak reduction, congestion avoidance, and voltage control.

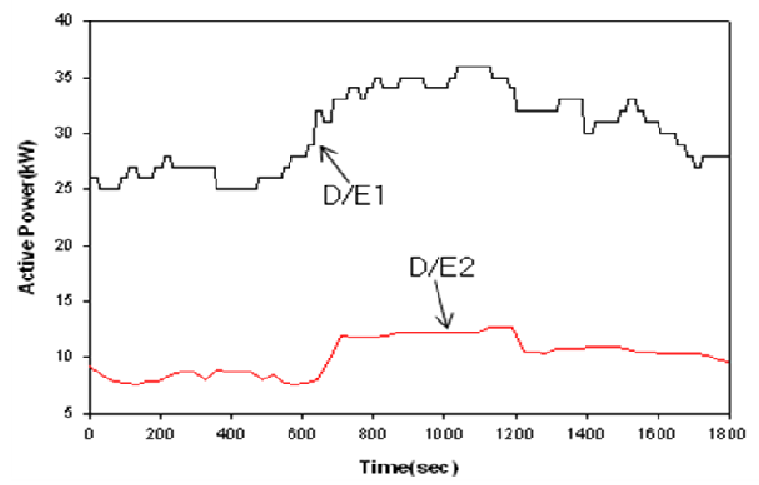

Fig. 12. Active power outputs of diesel engine generators. 


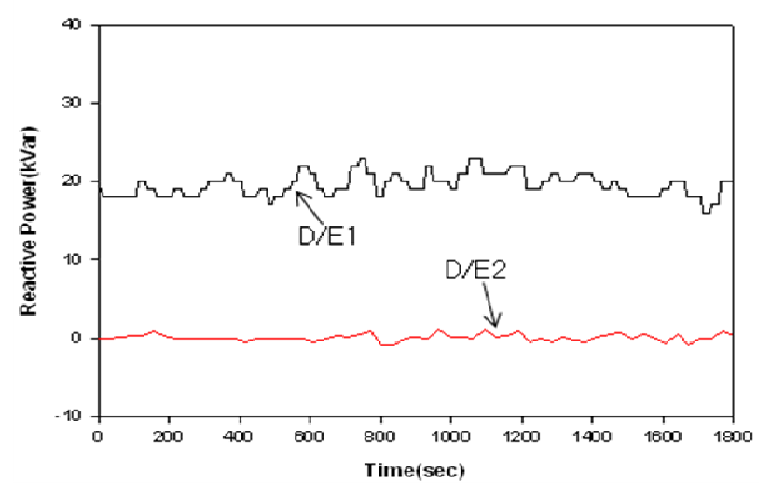

Fig. 13. Reactive power outputs of diesel engine generators.

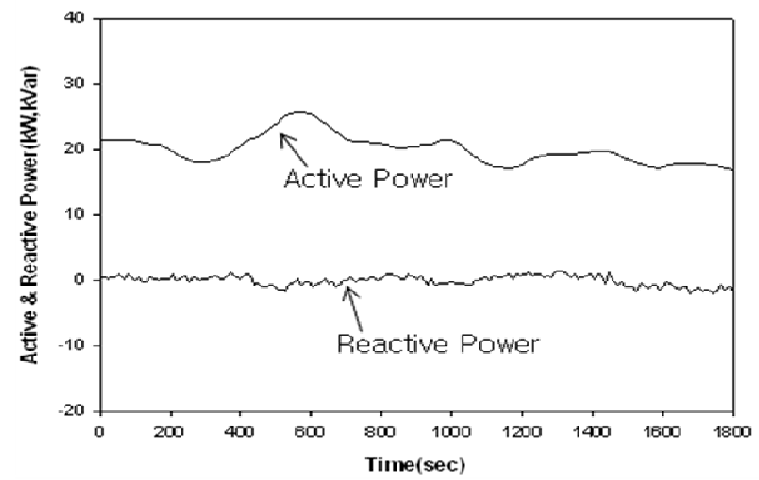

Fig. 14. Controlled power flow of PCC.

\section{B. Islanded Mode}

After islanding, the control scheme of BESS shifts to Frequency/Voltage control. As a result of the fast control acting of BESS, the frequency of the microgrid maintains within the range from $59.8 \mathrm{~Hz}$ to $60.2 \mathrm{~Hz}$ and the voltage also can be regulated from $0.9 \mathrm{pu}$ to $1.1 \mathrm{pu}$ during islanded operation as shown in Fig. 15 and Fig. 16. The power output of BESS changes from zero to a certain value to control the frequency and the voltage, and it is returned to zero due to secondary regulation control as shown in Fig. 17. During islanded operation, the power outputs of diesel engine generators are also changed from an initial constant value to a new set point as shown in Fig. 18 and Fig. 19. The results show that the proposed cooperative control scheme can regulate the frequency and voltage and reduce the consumption of the stored energy of ESS.

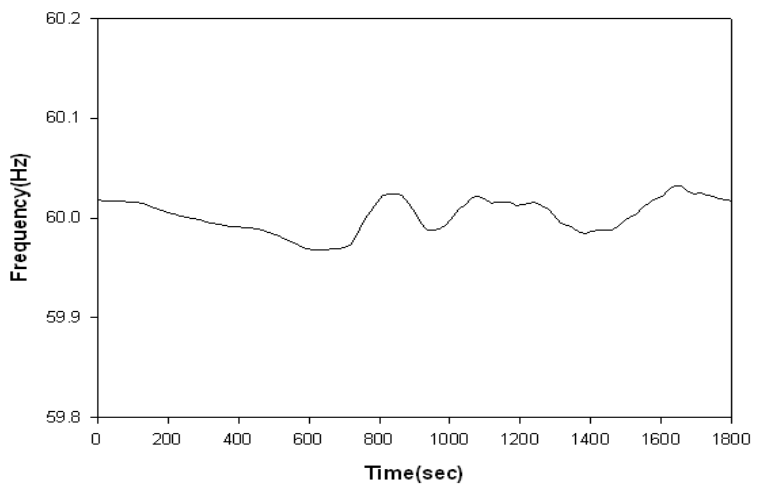

Fig. 15. Frequency of microgrid.

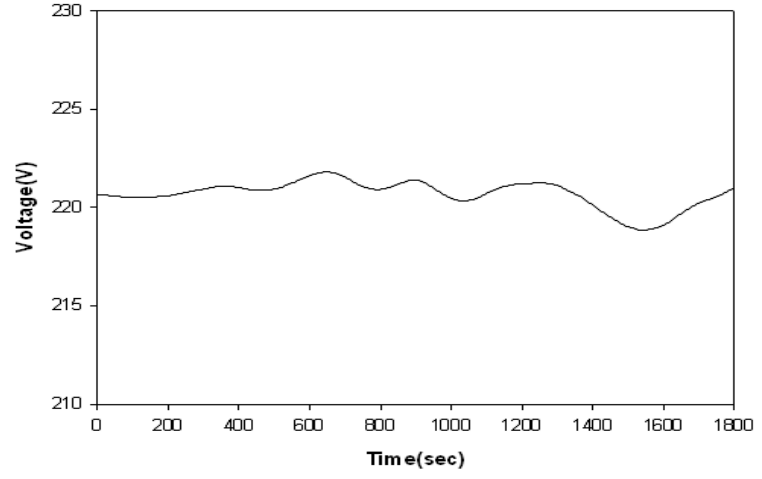

Fig. 16. Voltage of microgrid.

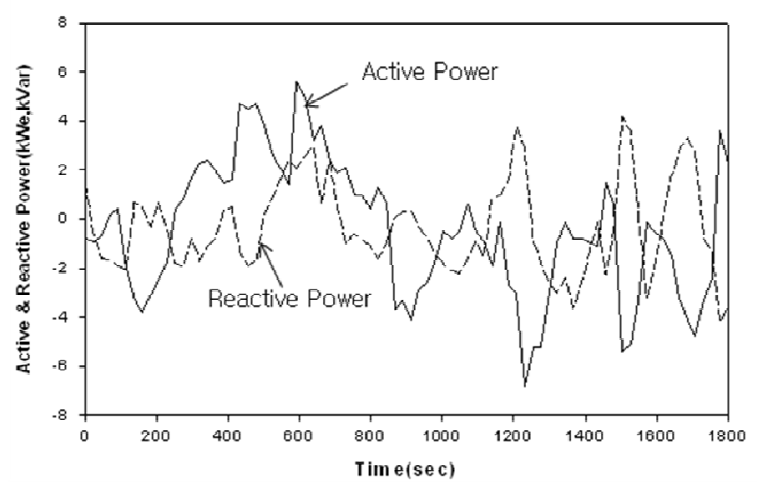

Fig. 17. Power output of BESS.

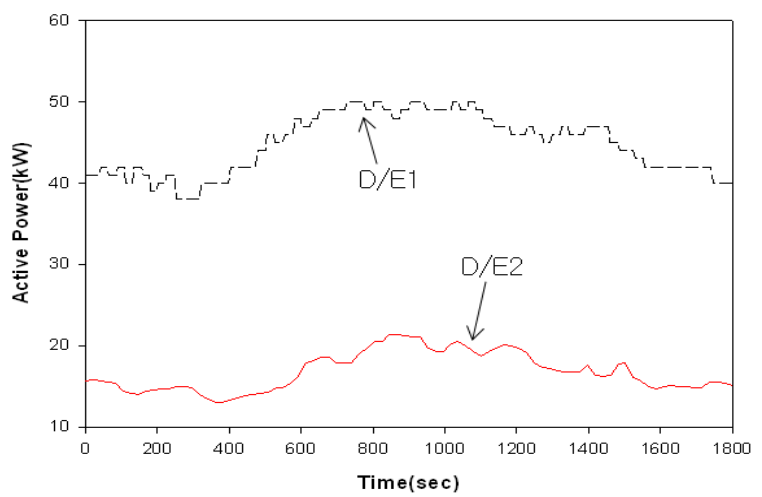

Fig. 18. Active power outputs of diesel engine generators.

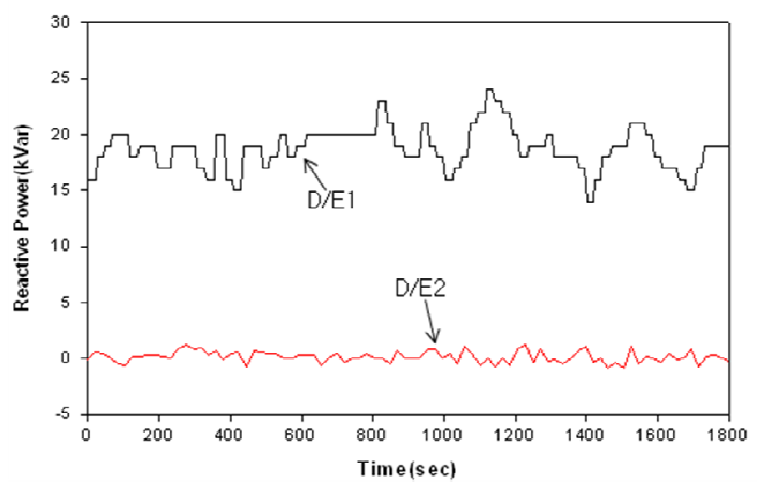

Fig. 19. Reactive power outputs of diesel engine generators.

\section{Conclusion}

In this paper, the management functions of MMS in microgrid are presented and evaluated by a hardware test using microgrid pilot plant. In grid-connected mode, the 
power flow at PCC is regulated at certain constant level by control algorithm of MMS. The test results show that microgrid can acts as a aggregated constant load or generator. This means that the distribution operator can utilize the microgrid to achieve the effective system operation goals such as peak reduction, congestion avoidance, and voltage control. In islanded mode, the ESS, which has a relatively fast response time, plays a primary control role. The test results indicate that the ESS can handle the frequency and the voltage very well. The control capability of the ESS may be limited, however, by its available energy storage capacity. Therefore, power output of ESS should be brought back to zero as soon as possible. The secondary regulation control is in charge of bringing back the current power output of ESS to a pre-planned value, usually zero. The results show that the proposed cooperative control scheme can regulate the frequency and voltage and reduce the consumption of the stored energy of ESS.

\section{References}

[1] CERT, "Integration of Distributed Energy Resources: The CERTS MicroGrid Concept”, LBNL-50829 ( 2003).

[2] N. D. Hatziargyriou, A.P. Meliopoulos, "Distributed Energy Sources: Technical Challenges", IEEE Power Engineering Society Winter Meeting 2002, pp. 1017-1022.

[3] F. Katiraei, M. R. Iravani, P. W. Lehn, "Micro-Grid Autonomous Operating During and Subsequent to Islanding Process", IEEE Trans. Power Delivery 2005, vol. 20, pp. 248-257.

[4] EU Microgrid Project, "Microgrids Large Scale Integration of Microgeneration to Low Voltage Grids", ENK-5-CT2002-00610 (2004).

[5] R.H. Lasseter, "MicroGrids", IEEE Power Engineering Society Winter Meeting 2001, pp. 146-149.

[6] Paolo Piagi, Robert Lasseter, "Autonomous Control of Microgrids", IEEE Power Engineering Society Meeting 2006.

[7] J. A. Pecas Lopes, C. L. Moreira, A. G. Madureira, "Defining Control Strategies for MicroGrids Islanded Operation", IEEE Trans. Power Syst. 2006, vol. 21, pp. 916-924. 